\title{
Editorial
}

\section{Distributed Control and Estimation of Networked Agent Systems}

\author{
Wenwu Yu, ${ }^{1}$ Ming Cao, ${ }^{2}$ Guanrong Chen, ${ }^{3}$ Guanghui Wen, ${ }^{4}$ and Xinghuo $\mathrm{Yu}^{5}$ \\ ${ }^{1}$ Department of Mathematics, Southeast University, Nanjing 210096, China \\ ${ }^{2}$ Faculty of Mathematics and Natural Sciences, ITM, University of Groningen, 9747 AG Groningen, The Netherlands \\ ${ }^{3}$ Department of Electronic Engineering, City University of Hong Kong, Hong Kong \\ ${ }^{4}$ School of Engineering and Information Technology, The University of New South Wales at ADFA, Canberra, ACT 2600, Australia \\ ${ }^{5}$ School of Electrical and Computer Engineering, RMIT University, Melbourne, VIC 3001, Australia
}

Correspondence should be addressed to Wenwu Yu; wenwuyu@gmail.com

Received 24 September 2013; Accepted 24 September 2013

Copyright (C) 2013 Wenwu Yu et al. This is an open access article distributed under the Creative Commons Attribution License, which permits unrestricted use, distribution, and reproduction in any medium, provided the original work is properly cited.

This special issue focuses on distributed control and estimation of networked agent systems. It is well known that, nowadays, most of the real-world large-scale systems can be modeled as networked agent systems, where examples include biological systems, multivehicle systems, and distributed sensor systems. Within this context, centralized control laws or estimation algorithms may be no longer valid since the size of such a networked agent system can be huge. Furthermore, each individual agent in such a networked system may have limited computational and sensing abilities; thus, distributed control and estimation design become necessary but challenging. Recently, distributed control and estimation of networked agent systems have been widely applied in various domains including distributed computing, formation control, spacecraft control, distributed sensor networks, and smart grids.

This special issue contains twenty-seven papers, most of which are related to distributed control and estimation of networked agent systems. In particular, six papers discuss coordination control for networked agent systems with communication constrains and higher-order dynamics. There are seven papers investigating synchronization in complex networks. In addition, modeling, identification, and optimization of networked agent systems are studied in another set of three papers. Furthermore, dynamics and control of networked systems are discussed in five papers. To apply dynamics and control of networked systems into some practical problems, six papers studied some potential applications in social network, water quality, UAVs, and so on.
It should be noted that the selected topics and published papers in this special issue are not a comprehensive study in distributed control and estimation of networked agent systems. However, they do provide some recent advances covering many topics in this field, which could benefit the current research to some extent.

\section{Wenwu Yu}

Ming Cao

Guanrong Chen

Guanghui Wen

Xinghuo Yu 


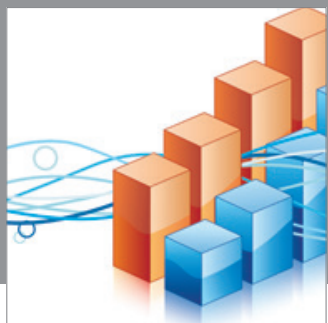

Advances in

Operations Research

mansans

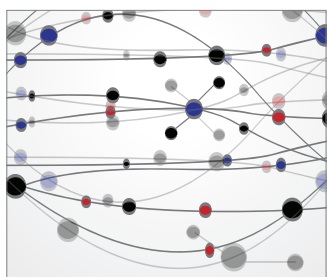

The Scientific World Journal
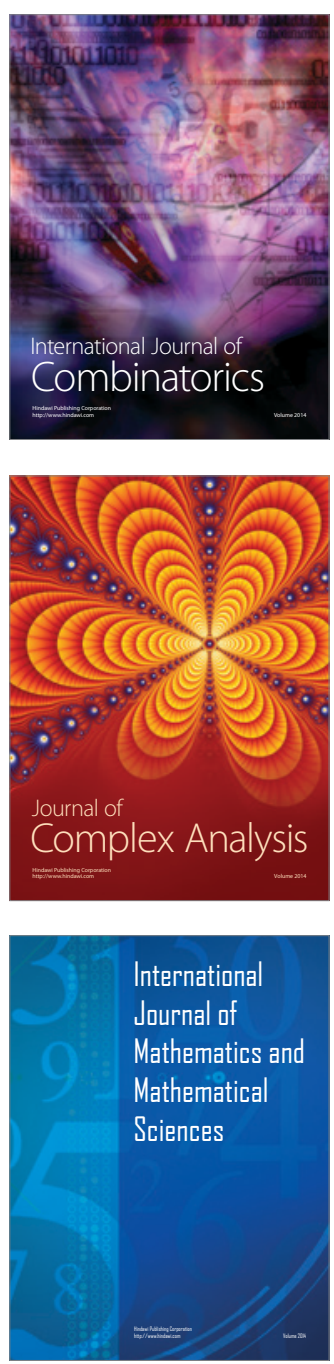
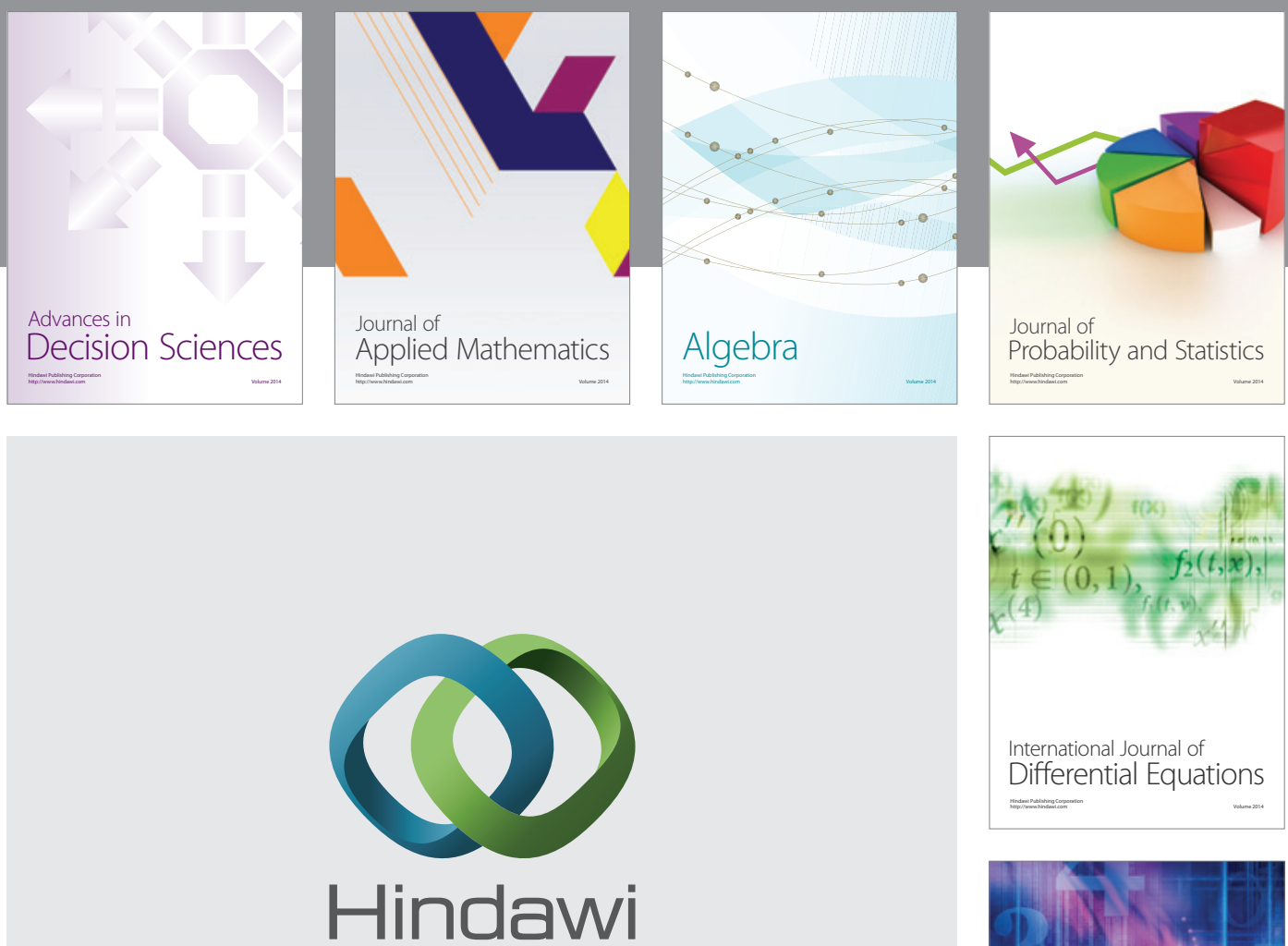

Submit your manuscripts at http://www.hindawi.com
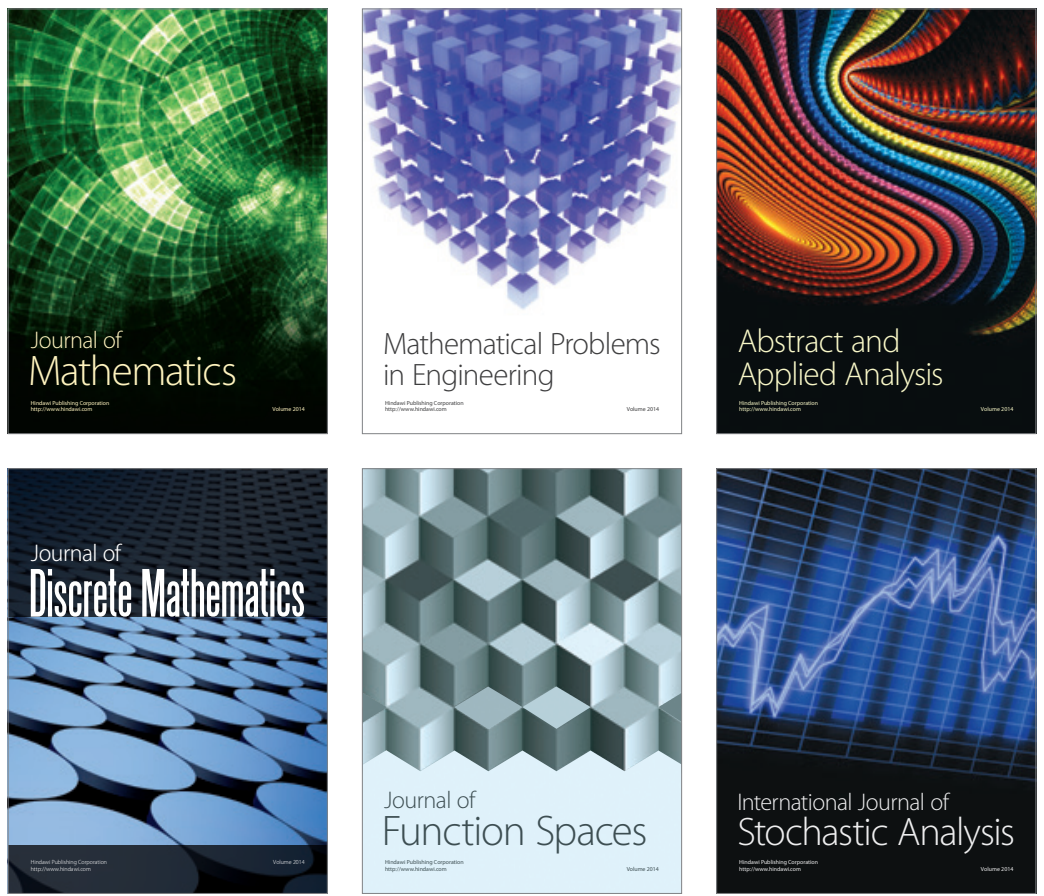

Journal of

Function Spaces

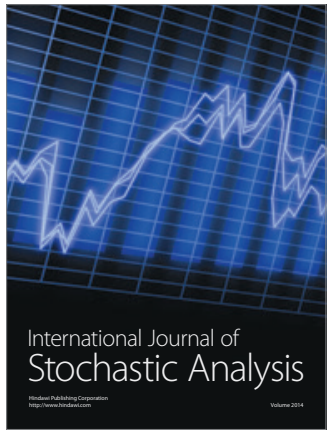

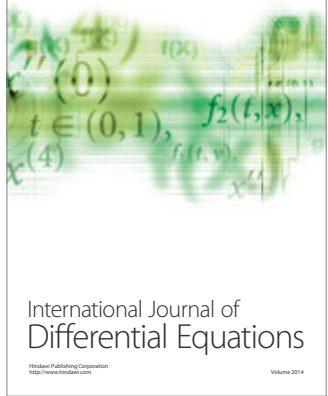
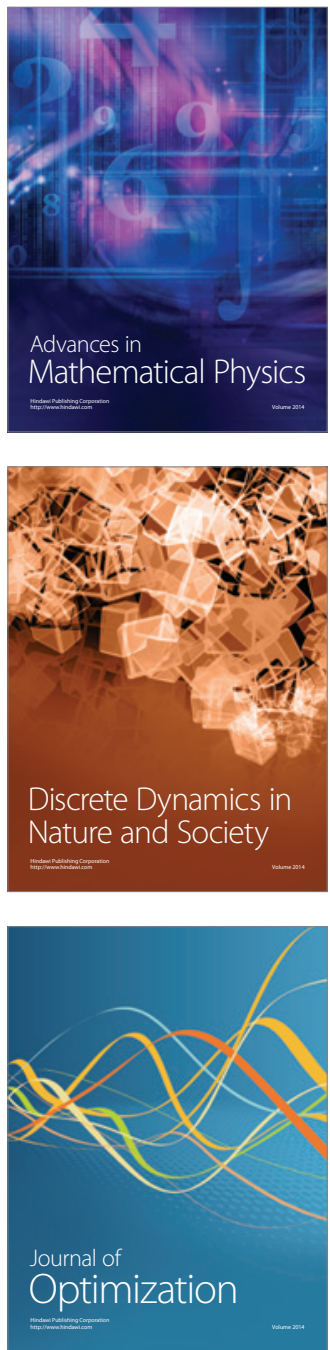